\title{
Comparison of Single Voxel and Multi Voxel Magnetic Resonance Spectroscopy in Evaluation of Brain Tumors
}

\author{
Anith Alfred J1, Abubacker Sulaiman F², Divya Shree ${ }^{3}$, Ashraf Ahmed ${ }^{4}$, Saravanan K ${ }^{5}$, Vijay Prabhu ${ }^{6}$ \\ ${ }^{1}$ Final Year Post Graduate Student, ${ }^{2}$ Professor, ${ }^{3}$ B.Sc Allied Health Science Student, ${ }^{4}$ Associate Professor, ${ }^{5}$ Final Year Post \\ Graduate Student, ${ }^{6}$ Assistant Professor, Department of Radiology and Imaging Sciences, Chettinad Hospital and Research \\ Institute, Kelambakkam, Kanchipuram-603103, Tamil Nadu, India.
}

Corresponding Author: Prof. Dr. Abubacker Sulaiman F, Professor Department of Radiology and Imaging sciences, Chettinad Hospital and Research Institute, Kelambakkam, Kanchipuram-603103, Tamil Nadu, India.

DOI: 10.21276/ijcmsr.2018.3.2.9

How to cite this article: Anith Alfred J, Abubacker Sulaiman F, Divya Shree, Ashraf Ahmed, Saravanan K, Vijay Prabhu. Comparison of Single Voxel and Multi Voxel Magnetic Resonance Spectroscopy in Evaluation of Brain Tumors. International Journal of Contemporary Medicine Surgery and Radiology. 2018;3(1):B36-B40.

\section{A B S T R A C T}

Introduction: Magnetic Resonance Spectroscopy is an advanced and non-invasive technique. Spectroscopic imaging or chemical shift imaging the data is converted as an image with the signal intensity representing the particular metabolites and its concentration. To assess the efficacy of Magnetic Resonance spectroscopy in characterizing the intracranial tumors, comparing single voxel and multi voxel $1 \mathrm{H}$ MR Spectroscopy.

Material and Methods: MRI findings suggestive of intra-cranial tumors were selected for the study and both single voxel and multi voxel MRS were done.

Results: Out of the total number of patients examined using single and multi voxel MRS, false peak values were more when using single voxel MR spectroscopy than when using multi voxel MR spectroscopy.

Conclusions: The use of multi voxel spectroscopy gave accurate analysis of the region of interest (ROI) in cases which may involve a tumor associated with hemorrhage / calcification / necrosis. While single voxel MRS, the operation is faster and easier, when compared to that of multi voxel MRS.

Key Words: Magnetic Resonance Imaging, Aminobutyric Acid, Glutamine, Single Voxel Spectroscopy

\section{INTRODUCTION}

Magnetic Resonance Spectroscopy is an advanced and noninvasive technique and has a potential for providing the information about the composition of various lesions. It is a useful method for determining the molecular structures which provides the metabolic information from viable brain tissues. ${ }^{1-3}$ With the development of improved techniques for spatial localization and water suppression (MRS) provides metabolic information regarding the tissue under study, complementing the anatomic information obtained with conventional MRI. For a reliable MRS procedure, spectroscopic localization methods and data acquisition should be properly adjusted. ${ }^{4-6}$ Metabolites which can be detected in the brain tissue include Choline, Creatinine, $\mathrm{N}$-acetyl aspartate (NAA), lactate, myoinositol, glutamine/ glutamate, lipids, and amino acids. Brain lesions contain abnormal levels of these metabolites as compared to normal brain tissue. ${ }^{7,8}$ Single-voxel MR spectroscopy measures the MR signal of a single selected region of interest whereas signal outside this area is suppressed. In single-voxel MRS, the magnetic field and the various other parameters are optimized to get the best possible spectrum from a relatively small region of the brain. ${ }^{9}$ Spectroscopic imaging or chemical shift imaging the data is converted as an image with the signal intensity representing the particular metabolites and its concentration. In a normal MR spectroscopy, the spectra obtained from the white matter and grey matter shows the predominant metabolites which are represented from right to left sequentially they are arranged as $\mathrm{N}$-acetyl aspartate, creatinine, choline and Myoinositol. ${ }^{10-}{ }^{12}$ Hence; we planned the present study to assess the efficacy of Magnetic Resonance spectroscopy in characterizing the intracranial tumors, comparing single voxel and multi voxel $1 \mathrm{H} M R$ Spectroscopy.

\section{MATERIAL AND METHODS}

30 patients with MRI findings suggestive of intra-cranial tumors were selected for the study. The patient with all age groups was included in this study. Patients undergoing MRI brain found to have intracranial tumors where MRS is applicable.

\section{Exclusion criteria}

- Patients on cardiac pacemakers.

- Metallic implants.

- Claustrophobic patients.

- Early pregnancy

A 1.5 Tesla SIGMA GE HDxt MRI scanner was used to scan all patients with a 8 channel NV (NAVIGATOR) 
radiofrequency coil was used. The bore size of the machine is $60 \mathrm{~cm}$. All the cases were examined in supine position with standard circularly polarized head coil using the following sequences. In this study all the patient have undergone routine sequence like diffusion weighted images (DWI) axial, T2 FLAIR axial, T2 TSE axial,T1 SE axial, T2 FLAIR coronal, T1 SE sagittal, T1 SE fat-sat pre-contrast axial, T1 SE fat-sat post contrast axial /sagittal /coronal and in addition (Figure 1). Single voxel and Multi-voxel spectroscopy will be used in all studies. Minimum of two areas will be selected for the study.

To get an accurate assessment of the tumor chemistry, the spectroscopic voxel were placed over an enhancing region of the tumor, avoiding areas of necrosis, hemorrhage, calcification, or cysts. Point Resolved Spectroscopy (PRESS) is the sequence used in single voxel and multi voxel spectroscopy. It employs one 900 and two 1800 slice selective pulses along each of the spatial direction, generating signals in the form of a spin echo.

\section{Single voxel spectroscopy}

When short echo times are selected, single voxel MRS performs more vividly. ${ }^{11}$ By employing a short echo time, it provides a high signal to noise ratio spectra and minimizes the loss of signal due to fast decaying peaks of metabolites (Figure 2). Hence for single voxel examination short echo time PRESS with TE less than $35 \mathrm{~ms}$ is preferred.

In chemical shift imaging (CSI), typically more than 100 spectra per slice or volume are simultaneously acquired. A selective RF pulse is used for slice selection. All spatial information is encoded into the phase of MRI with the help of CSI. Here, acquisition of data is performed without frequency encoding gradient to retain chemical shift information. Through phase encoding step, many spectra from a slice or a $3 \mathrm{D}$ volume can be simultaneously acquired. This imaging is combined with PRESS but only selecting a larger volume than for a single voxel MRS (Figure 3).

\section{Signal to Noise Ratio}

SNR is the ratio between amplitude of resonance received to the average amplitude of the noise. The noise is the frequency that is random in space and time. It is constant for all patients and is primarily dependant on the built of the patient or the inherent noise of the system. Region of interest (ROI) was carefully placed to avoid strong interference from subcutaneous fat and lipids of the skull, and outer volume suppression (OVS) slabs outside the ROI was used to further reduce the potential for artifact.

\section{Analysis of the spectroscopic data}

The main metabolites identified by MRS are (NAA) at 2.02 $\mathrm{ppm},(\mathrm{Cr})$ at $3.0 \mathrm{ppm}$, (Cho) at $3.2 \mathrm{ppm}$ and $(\mathrm{mI})$ at 3.6 $\mathrm{ppm}$. Concerning lipids and lactate we qualitatively defined and estimated their sum (LL) between 0.9 and $1.3 \mathrm{ppm}$. The following metabolite ratio was calculated using the standard commercial software: Cho/NAA, Cho+Cr/NAA, $\mathrm{Cho} / \mathrm{Cr}, \mathrm{LL} / \mathrm{Cr}$ and $\mathrm{NAA} / \mathrm{Cr}$ in addition to $\mathrm{MI} / \mathrm{Cr}$ in the second technique when the short TE has been used. In case of 2DMRSI, only voxels presenting the highest Cho/NAA, $\mathrm{Cho}+\mathrm{Cr} / \mathrm{NAA}$, Cho/Cr, $\mathrm{LL} / \mathrm{Cr}$ and the lowest NAA/Cr in the solid part of the tumor were considered. A spectrum was excluded for analysis if integration of any peak could not be accomplished using the automated analysis software.

\section{RESULTS}

A total of 30 patients were examined. Metabolites ratio is shown in Figure 4. In 8 patients, false peak value occurred. Out of the total number of patients examined using single and multi voxel MRS, a few number of patients show a false peak value when using single voxel MR spectroscopy whereas the same was not true when using multi voxel MR spectroscopy (Figure 5). Majority of the patients in the present study belonged to the age group of 40 to 70 years (Figure 6). Among these 30 patients, 60 percent of them were males while the remaining were females (Figure 7). In using MRS for application to brain tumors, the limited spatial resolution (usually $1 \mathrm{~cm} 3$ or less for MRSI and $\sim 4$ to 8 $\mathrm{cm} 3$ for single voxel MRS) and partial volume effects should be kept in mind. For example, in treatment planning, MRSI pixel whose 'point-spread function' overlaps both tumor and normal tissue has the potential to be mis-classified as tumor invasion into surrounding brain if this issue is neglected. For this reason, use of high as possible spatial resolution is recommended. High resolution MRSI with good signal-

\begin{tabular}{|c|c|c|c|c|c|c|c|c|}
\hline \multirow[t]{2}{*}{ Parameters } & \multirow[t]{2}{*}{ T2 flair } & \multirow[t]{2}{*}{ T2 fse } & \multirow[t]{2}{*}{ T1 se } & \multirow[t]{2}{*}{ T2 flair } & \multirow[t]{2}{*}{ T1 se } & \multirow[t]{2}{*}{ T1 se } & \multicolumn{2}{|c|}{ MRS } \\
\hline & & & & & & & single & multi \\
\hline Acquisition Method & $2 \mathrm{~d}$ & $2 \mathrm{~d}$ & $2 \mathrm{~d}$ & $2 \mathrm{~d}$ & $2 d$ & $2 \mathrm{~d}$ & MRS & MRS \\
\hline Plane & axial & axial & axial & Coronal & sagittal & axial & axial & axial \\
\hline FOV & 24 & 24 & 24 & 28 & 26 & 24 & 24 & 24 \\
\hline TR & 6500 & 4000 & 800 & 6500 & 835 & 830 & 1500 & 1000 \\
\hline $\mathrm{TE}$ & 800 & 650 & 75 & 800 & 80 & 90 & 35 & 144 \\
\hline Matrix & $320 \times 244$ & $320 \times 256$ & $320 \times 192$ & $320 \times 224$ & $320 \times 192$ & $320 \times 224$ & $1 \times 1$ & $16 \times 16$ \\
\hline NEX & 2 & 2 & 2 & 1 & 2 & 1 & 8 & 1 \\
\hline FAT-SAT & NO & NO & NO & NO & NO & YES & - & - \\
\hline Slice Thickness & 4 & 4 & 4 & 4 & 4 & 4 & - & - \\
\hline Voxel Thickness & - & - & - & - & - & - & 20 & 40 \\
\hline Inter slice & 1 & 1 & 1 & 1 & 1 & 1 & - & - \\
\hline Timing (min) & 3.28 & 1.45 & 1.05 & 3.28 & 1.15 & 3.45 & 3.00 & 4.2 \\
\hline IV Contrast & NO & NO & NO & NO & NO & YES & - & - \\
\hline
\end{tabular}




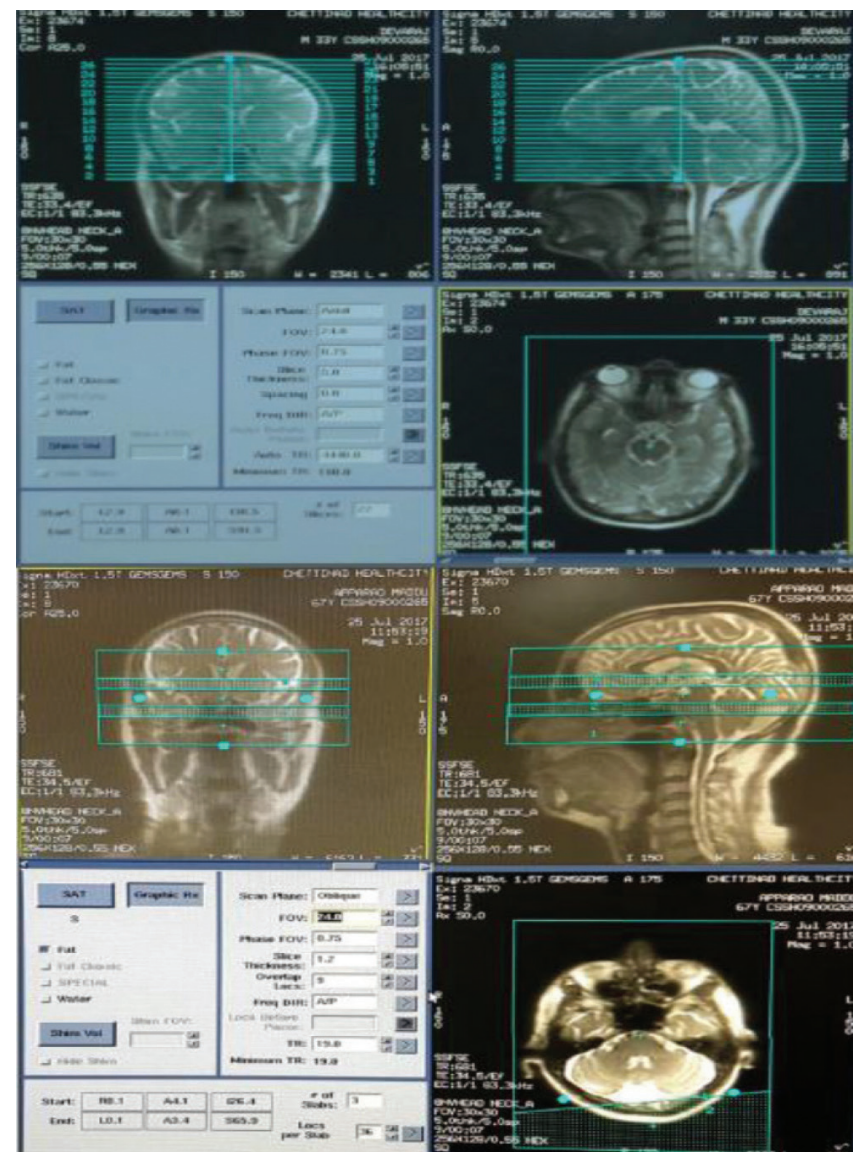

Figure-1: Planning of axial plane

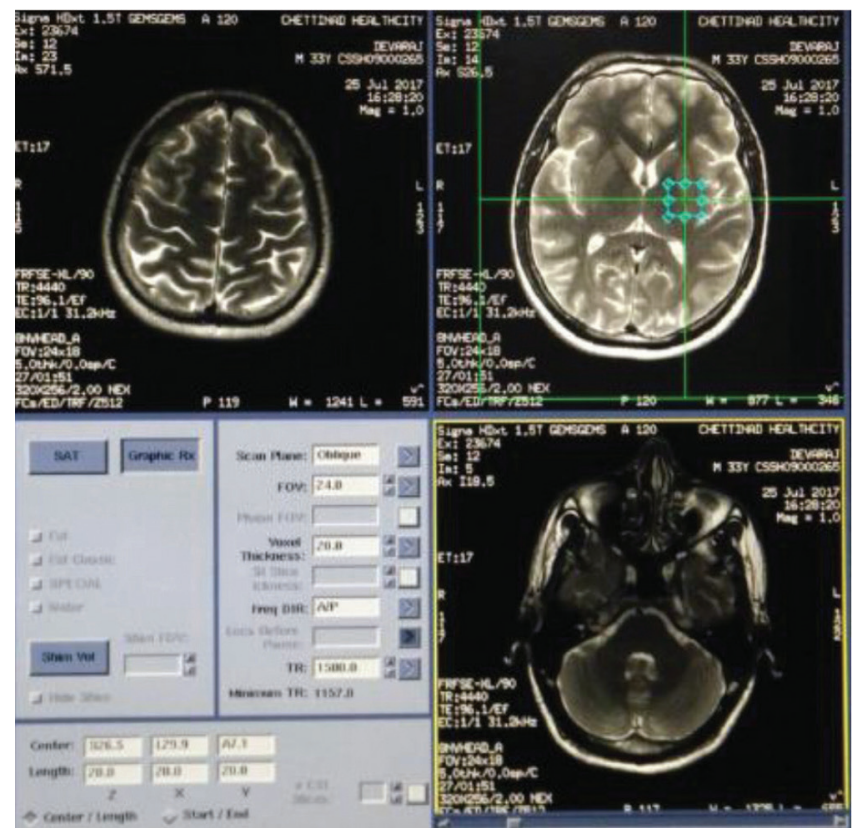

Figure-2: Planning of single voxel spectroscopy

to-noise ratios is best performed using high magnetic field strengths and multi-channel phased-array coils, and efficient pulse sequences. Both single and multi voxel MRS was applied to the total number of patients examined. Hence the use of multi voxel spectroscopy is suggested to give accurate analysis of the region of interest (ROI) in cases which may involve a tumor associated with hemorrhage / calcification / necrosis.

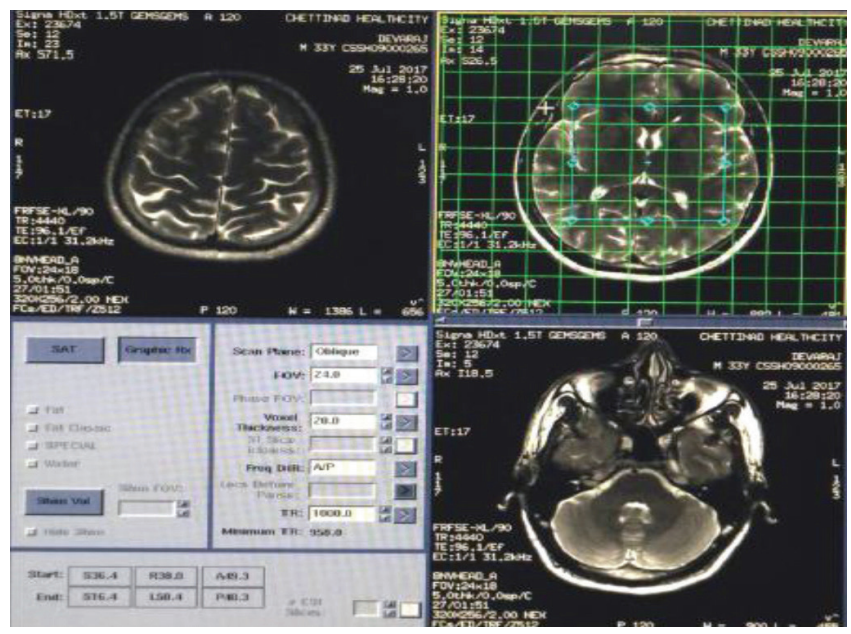

Figure-3: Planning of multi voxel spectroscopy

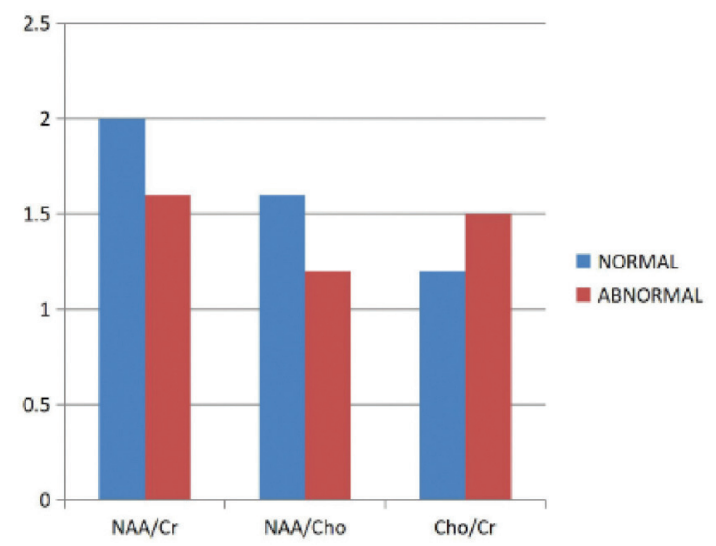

Figure-4: Metabolite ratios

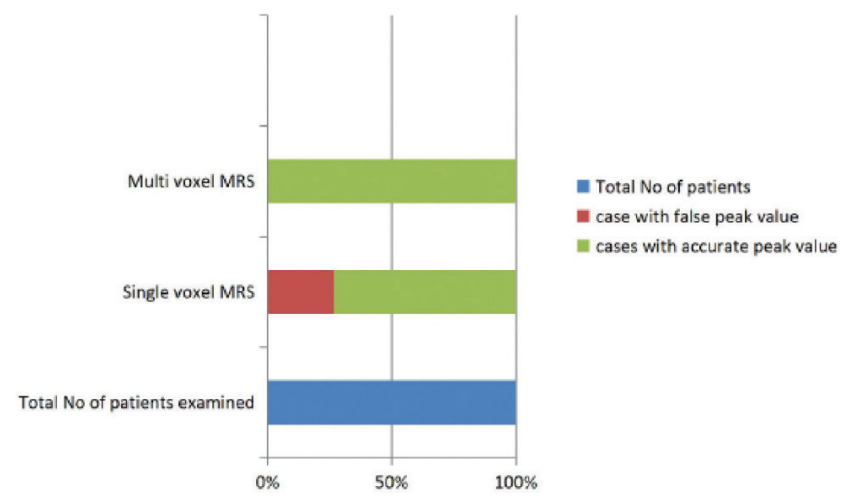

Figure-5: Accurate vs false peak value

\section{DISCUSSION}

When performing the single voxel spectroscopy we employed a short TE technique in order to make initial diagnosis since all the metabolites represented have a high SNR. When performing the multi voxel spectroscopy a long TE is employed to characterize different regions of lesion/mass in order to assess the parenchyma of the brain both around and adjacent to the mass/lesion. This method is used to assess response to therapy and to aid the prognosis in search of tumor recurrence.

The Hunter's angle is a common way to analyze the chemical spectra while looking for metabolite ratios, namely 


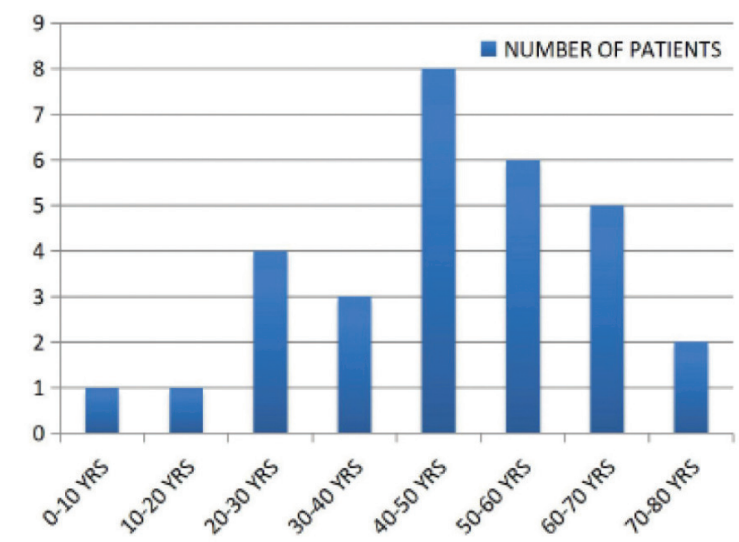

Figure-6: No of patients

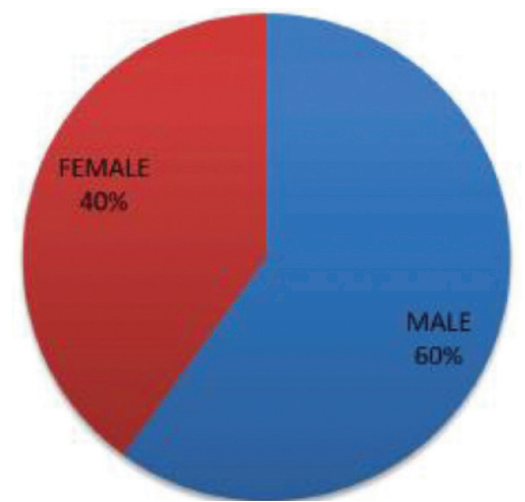

Figure-7: Gender demography

NAA: Cr; NAA: Cho; Cho: Cr. With the help of a known reference solution from the MR spectral data an absolute concentration of metabolite can be calculated. A single voxel MRS, the operation is faster and easier, when compared to a multi voxel MRS.A limited volume of interest in a single voxel MRS allows an excellent shim as opposed to a multi voxel MRS where it is difficult to shim over the total area of interest. There is excellent spectral quality and peak separation with high signal to noise quantification in case of a single voxel MRS when compared to a multi voxel MRS which shows lower signal to noise and poses difficulties with quantification. The spectral contamination in case of a single voxel MRS is due to partial volume and chemical shift displacement effects from adjacent tissues. In a multi voxel MRS, the chemical shift aliasing is due to the bleeding of spectra from the adjacent voxel. A single voxel consumes about 3-5 minutes per voxel and hence is quicker compared to amulti voxel MRS which takes about 5-8 minutes for 2D imaging and 7-15 minutes for 3D imaging. ${ }^{12}$

In cases involving tumor associated with adjacent hemorrhage or calcification or necrosis, applying single voxel spectroscopy may give arise to a false peak value in the spectra because of the ROI being localized to the tumor region which also includes adjacent hemorrhage or calcification or necrosis.

Whereas in a similar case applying multi voxel spectroscopy is considered the best method to assess tumor region because of the ROI being individually and independently localized to the tumor and its constituents only, thus giving a true peak value in the spectra. ${ }^{13-16}$

Relatively common metabolic changes in human brain tumors are elevated signals in the lactate and lipid region of the spectrum, and also sometime increased levels of myo-inositol (mI) in short echo time (TE) spectra. ${ }^{17-19}$ The increase in lactate is most likely the result of anaerobic glycolysis, although it could also be due to insufficient blood flow leading to ischemia, or possibly also due to necrosis. ${ }^{20}$ Choi $\mathrm{S}$ et al measure the difference in levels of cerebral metabolites in the right and left hemispheres, gray (GM) and white matter (WM), imaging planes, and anatomical regions of healthy dogs to establish normal variations. There was no significant difference in levels of these metabolites between the right and left in any locations or between the GM and WM in the cerebral hemispheres. However, there were significant differences in metabolite ratios within imaging planes. The NAA/Cr was lower in the cerebellum than other regions and the thalamus had a higher $\mathrm{Cho} / \mathrm{Cr}$ and lower NAA/Cho ratio than in other regions. The spectral and metabolic values will provide a useful internal reference for clinical practice and research involving multi-voxel magnetic resonance spectroscopy. Measurement of metabolite values in the transverse plane is recommended for comparing levels of regional metabolites. ${ }^{15}$ Steffen-Smith EA et al determined the feasibility of two magnetic resonance spectroscopy techniques in pediatric patients with diffuse intrinsic pontine gliomas (DIPGs) and evaluate the relationship of metabolic profiles determined by each technique. Children with DIPG $(n=36)$ were evaluated using single voxel spectroscopy (SVS) and magnetic resonance spectroscopic imaging (MRSI) during the same imaging session. Patients were followed longitudinally ( $\mathrm{n}=150$ total studies). Technical feasibility was defined by sufficient water and lipid suppression for detection of metabolites. Correlation of metabolic data obtained by SVS and MRSI was determined using the Spearman rank method. Metabolite ratios, including Choline:N-acetylaspartate (Cho:NAA) and Cho:Creatine (Cho:Cr), were obtained from SVS and MRSI. SVS and MRSI acquisition were feasible in $>90 \%$ of studies. Maximum Cho:NAA and Cho:Cr from MRSI analysis were strongly associated with Cho:NAA and Cho:Cr obtained by SVS ( $\mathrm{r}=0.67$ and 0.76, respectively). MRSI Cho:NAA values were more heterogeneous compared to $\mathrm{Cho:Cr}$ values within the same lesion, and a strong linear relationship between the range and maximum Cho:NAA values was observed. SVS and MRSI acquisition was feasible, with a strong correlation in metabolic data. Both techniques may improve diagnostic evaluation and management of DIPG. SVS is recommended for global assessment of tumor metabolism before and after therapy. ${ }^{16}$

\section{CONCLUSION}

Hence, the application of single and multi voxel spectroscopy is solely dependent on the nature of the study and its application is employed uniquely when needed for different individuals depending on the characteristics of the tumor. However the use of multi voxel spectroscopy is highly suggested because of its flexible characteristics and accurate localization of ROI.

\section{REFERENCES}

1. Dowling C, Bollen AW, Noworolski SM, McDermott 
MW, Barbaro NM, Day MR, Henry RG, Chang SM, Dillon WP, Nelson SJ, Vigneron DB. Preoperative proton MR spectroscopic imaging of brain tumors: correlation with histopathologic analysis of resection specimens. American Journal of Neuroradiology. 2001;22(4):604-12.

2. Steinmetz H, Huang YX. Two-dimensional mapping of brain surface anatomy. American journal of neuroradiology. 1991;12(5):997-1000.

3. Morita N, Harada M, Otsuka H, Melhem ER, Nishitani H. Clinical application of MR spectroscopy and imaging of brain tumor. Magnetic Resonance in Medical Sciences.2010;9(4):167-75.

4. vanderGraafM.Invivo magneticresonancespectroscopy: basic methodology and clinical applications. European Biophysics Journal. 2010;39(4):527-40.

5. Bulik M, Jancalek R, Vanicek J, Skoch A, Mechl M. Potential of MR spectroscopy for assessment of glioma grading. Clinical neurology and neurosurgery. 2013;115(2):146-53.

6. Möller-Hartmann W, Herminghaus S, Krings T, Marquardt G, Lanfermann H, Pilatus U,Zanella F. Clinical application of proton magnetic resonance spectroscopy in the diagnosis of intracranial mass lesions. Neuroradiology. 2002;44(5):371-81.

7. Bottomley PA. Selective volume method for performing localized NMR spectroscopy. U.S. patent 1984;4:480,228.

8. Ordidge RJ, Bendall MR, Gordon RE, Connelly A. Volume selection for in vivo biological spectroscopy. In: Govil G, KhetrapalCL, Saran A, eds. Magnetic resonance in biology and medicine. New Delhi, India : McGraw Hill, 1985 ; 387 - 397.

9. Frahm J, Merboldt KD, Hänicke W. Localized proton spectroscopy using stimulated echoes. J Magn Reson 1987 ; 72 ( 3 ): 502- 508 .

10. Bruhn H, Frahm J, Gyngell ML, Merboldt KD, Hänicke W, Sauter R. Cerebral metabolism in man after acute stroke: new observations using localized proton NMR spectroscopy. Magn Reson Med 1989 ; 9 ( 1 ): $126-131$.

11. Gonal, J.S., \& Kohir, V.V. Classification of brain tumors by mining MRS spectrums using LabVIEW metabolite peak height scanning method. 2015 International Conference on Electrical, Electronics, Signals, Communication and Optimization (EESCO), 2015;16.

12. F. Abubacker Sulaiman, K.Saravanan, D. Raju.MR Spectroscopy Imaging in Paediatric Posterior Fossa Tumors.Poster No.: C-1023. Congress: ECR 2018.

13. Magudeeswaran PK, Farook AS, Ganesan P, Nataraja V, Rajamani A. A Vital Role of Magnetic Resonance Imaging in Pregnancy-Related Neurological Complications. Int J Sci Stud 2017;4(12):192-198.

14. J. Anith Alfred, F. Abubacker Sulaiman, K. Saravanan. MR Spectroscopy and Metabolic Imaging in Brain lesions.Poster No.: C-1657. Congress: ECR 2017.

15. Choi S, Song Y, Lee K, Lee Y, Choi H. Multi-voxel magnetic resonance spectroscopy of cerebral metabolites in healthy dogs at 1.5 Tesla. Journal of Veterinary Science. 2016;17(2):217-224.
16. Steffen-Smith EA, Venzon DJ, Bent RS, Hipp SJ, Warren KE. Single and multi-voxel proton spectroscopy in pediatric patients with diffuse intrinsic pontine glioma. International journal of radiation oncology, biology, physics. 2012;84(3):774-779.

17. Alger JR, Frank JA, Bizzi A, et al. Metabolism of human gliomas: assessment with $\mathrm{H}-1 \mathrm{MR}$ spectroscopy and F-18 fluorodeoxyglucose PET. Radiology. 1990;177(3):633-641.

18. Castillo M, Smith JK, Kwock L. Correlation of myoinositol levels and grading of cerebral astrocytomas. AJNR Am J Neuroradiol. 2000;21(9):1645-1649.

19. Herholz $\mathrm{K}$, Heindel $\mathrm{W}$, Luyten $\mathrm{PR}$, et al. In vivo imaging of glucose consumption and lactate concentration in human gliomas. Ann Neurol. 1992;31(3):319-327.

20. Hattingen E, Raab P, Franz K, et al. Myo-inositol: a marker of reactive astrogliosis in glial tumors? NMR Biomed. 2008;21(3):233-241.

Source of Support: Nil; Conflict of Interest: None

Submitted: 25-04-2018; Published online: 01-05-2018 